\title{
Lessons from the past: Long-term safety and survival outcomes of a prematurely terminated randomized controlled trial on prophylactic vs. hemoglobin-based administration of erythropoiesis-stimulating agents in patients with chemotherapy-induced anemia
}

\author{
GIANNIS MOUNTZIOS ${ }^{1}$, GERASIMOS ARAVANTINOS ${ }^{2}$, ZOI ALEXOPOULOU ${ }^{3}$, \\ ELENI TIMOTHEADOU ${ }^{4}$, FOTINI MATSIAKOU ${ }^{5}$, CHRISTOS CHRISTODOULOU ${ }^{6}$, KONSTANTINOS LASCHOS $^{2}$, \\ ELENI GALANI ${ }^{6}$, ANGELOS KOUTRAS $^{7}$, DIMITRIOS BAFALOUKOS $^{8}$, HELENA LINARDOU $^{8}$, \\ DIMITRIOS PECTASIDES ${ }^{9}$, IOANNIS VARTHALITIS ${ }^{10}$, PAVLOS PAPAKOSTAS ${ }^{5}$, \\ HARALAMBOS P. KALOFONOS $^{7}$ and GEORGE FOUNTZILAS ${ }^{4}$
}

\begin{abstract}
${ }^{1}$ Department of Medical Oncology, 251 Airforce General Hospital, 11525 Athens; ${ }^{2}$ Second Department of Medical Oncology, 'Agii Anargiri' Cancer Hospital, 14564 Athens; ${ }^{3}$ Health Data Specialists Ltd., 11526 Athens; ${ }^{4}$ Department of Medical Oncology, 'Papageorgiou' Hospital, Aristotle University of Thessaloniki School of Medicine, 56403 Thessaloniki; ${ }^{5}$ Oncology Unit, 'Hippokration' Hospital, 11527 Athens; ' ${ }^{6}$ Second Department of Medical Oncology, 'Metropolitan' Hospital, 18547 Piraeus;

${ }^{7}$ Division of Oncology, Department of Medicine, University Hospital, University of Patras Medical School, 26504 Patras; ${ }^{8}$ First Department of Medical Oncology, 'Metropolitan' Hospital, 18547 Piraeus; ${ }^{9}$ Oncology Section, Second Department of Internal Medicine, 'Hippokration' Hospital, 11527 Athens;

${ }^{10}$ Oncology Department, General Hospital of Chania, 73300 Chania, Greece
\end{abstract}

Received January 15, 2015; Accepted September 10, 2015

DOI: $10.3892 / \mathrm{mco} .2015 .693$

\begin{abstract}
Prophylactic erythropoiesis-stimulating agent (ESA) administration for chemotherapy-induced anemia (CIA) is not supported by current guidelines. Long-term follow-up of patients who had been treated with ESA for CIA in the past may provide useful information. In 2002, we undertook a prospective, randomized phase III trial of prophylactic vs. hemoglobin (Hb)-based (threshold: $11 \mathrm{mg} / \mathrm{dl}$ ) ESA administration in patients with solid tumors and CIA. ESA administration for CIA was permanently suspended in 2007 in view of published data at that time, while patient surveillance continued. Among 630 evaluable patients, $38.6 \%$ were male, $50.9 \%$ had advanced cancer at diagnosis, $40.6 \%$ had $\mathrm{Hb}$ levels $<12 \mathrm{mg} / \mathrm{dl}$ at baseline and $47.9 \%$ received ESA prophylactically (1:1 randomization). The major tumor types included colorectal $(36.0 \%)$, breast $(20.6 \%)$, non-prostate genitourinary $(11.0 \%)$ and lung cancer $(8.4 \%)$.
\end{abstract}

Correspondence to: Dr Giannis Mountzios, Department of Medical Oncology, 251 Airforce General Hospital, 3 P. Kanellopoulou Avenue, 11525 Athens, Greece

E-mail: gmountzios@gmail.com; hecogoff@otenet.gr

Key words: stroke, solid tumors, chemotherapy-induced anemia, erythropoietin, safety, toxicity, efficacy, thrombosis, pulmonary embolism, myocardial infarction
After a median follow-up of 85.4 months, 358 patients had relapsed and 380 had succumbed to the disease. Patients in the prophylactic ESA group (group A; experimental arm), as compared with those in the Hb-based group (group B; iron supplementation alone), exhibited a significantly more prominent increase in median $\mathrm{Hb}$ levels, particularly in the subset of patients with non-metastatic disease (two-sided $\mathrm{P}<0.01$ ) Among patients receiving chemotherapy for advanced cancer, those who received ESAs prophylactically exhibited a lower incidence of CIA (all grades: $\mathrm{P}=0.014$, grades $3-4$ : $\mathrm{P}=0.034$ ) and fatigue (all grades: $\mathrm{P}<0.001$, grades $3-4$ : $\mathrm{P}=0.055$ ), but a higher rate of a composite outcome encompassing all thrombosis-related events (all grades: $\mathrm{P}=0.043$, grades 3-4: $\mathrm{P}=0.099$ ). These differences were less prominent in the group of patients who received adjuvant treatment. There were no significant differences in overall mortality and relapse/progression rates between the two groups. Therefore, prophylactic, compared with $\mathrm{Hb}$-based, administration of ESAs for CIA in patients with solid tumors, was found to be associated with a significantly lower incidence of anemia and fatigue, but with a marginally higher rate of thrombosis-related adverse events, particularly in patients receiving first-line chemotherapy for advanced cancer.

\section{Introduction}

Anemia is prevalent in 30-90\% of cancer patients (1) and may be associated with either the malignancy and its complications, 
or the myelosuppressive effect of anticancer treatment, particularly chemotherapy. Correction of anemia may be achieved by either treating the underlying etiology, or by providing supportive care by transfusion with packed red blood cells (PRBCs) and administration of erythropoiesis-stimulating agents (ESAs), such as epoetin $\alpha$, epoetin $\beta$ and darbepoetin $\alpha$, with or without iron supplementation. The latter strategy is widely used in patients who develop chemotherapy-induced anemia (CIA) due to the suppressive effect of cytotoxic chemotherapeutic agents on precursors of the erythroid lineage (2). In this setting, clinical studies and subsequent meta-analyses (3-5) have found that ESAs increase hemoglobin $(\mathrm{Hb})$ levels and reduce the need for blood transfusions in cancer patients, with a positive effect on the overall quality of life.

Following a 'popularity' peak of ESAs among the global medical community in 2003-2004, in 2007 the Federal drug Association (FDA) of the United States issued substantial revisions to the label information and regulations regarding epoetin and darbepoetin $\alpha$, including addition of a 'Black Box' label warning (available online at http://www.medscape.com/viewarticle/553499). These amendments were based on the results of numerous randomized studies that individually demonstrated a decrease in overall survival (OS) and/or decreased locoregional disease control with ESA usage for advanced breast, cervical, head and neck, lymphoid, and non-small-cell lung cancer, as well as an increased risk of thrombosis-related events (2-4,6-8). A common characteristic in all these trials was an off-label target $\mathrm{Hb}$ level of $>12 \mathrm{mg} / \mathrm{dl}$, which prompted FDA to discourage prophylactic erythropoietin administration above this threshold. In view of these data, the European Society for Medical Oncology (ESMO) (http://annonc.oxfordjournals. org/content/21/suppl_5/v244.full.pdf), the American Society for Clinical Oncology (http://www.asco.org/quality-guidelines/ asco-ash-clinical-practice-guideline-update-use-epoetin-anddarbepoetin-adult), as well as the National Comprehensive Cancer Network guidelines (https://www.nccn.org/store/login/ login.aspx?ReturnURL=http://www.nccn.org/professionals/ physician_gls/pdf/anemia.pdf), currently do not recommend the prophylactic use of ESAs in patients with advanced cancer, or the use of ESAs for patients who receive chemotherapy with a curative intent, independently of the $\mathrm{Hb}$ levels.

In 2002, in view of the data available at that time, we undertook a prospective, randomized, controlled trial to evaluate the safety and efficacy of prophylactic ESA administration in patients with CIA, as compared to Hb-based ESA administration. Patients with either curable or advanced solid tumors were randomly assigned to receive erythropoietin prophylactically (i.e., independently of the $\mathrm{Hb}$ levels prior to the initiation of chemotherapy), or based on Hb levels (only patients with $\mathrm{Hb}$ levels $<11 \mathrm{mg} / \mathrm{dl}$ during chemotherapy received erythropoietin). In 2007 , following the enrolment of 630 patients, the Independent Safety and Data Monitoring Committee decided to discontinue the trial in view of the FDA recommendation published that year; further accrual was permanently suspended, while patients who had already been randomized continued to be under medical surveillance by the study investigators. In view of recent data and guidelines, we hypothesized that patients in the prophylactic ESA administration arm may be at an increased risk for thrombosis-related adverse events, disease relapse or progression and death. Herein, we provide long-term outcomes regarding safety, efficacy and mortality in the two patient groups after $>7$ years of follow-up.

\section{Patients and methods}

Study protocol. The HE 30/02 trial was an open-label, randomized, multicenter, phase III trial of prophylactic vs. Hb-based ESA administration in patients with solid tumors. Eligible patients were required to have a histologically or cytologically confirmed solid malignancy, a scheduled chemotherapy duration of $\geq 3$ months and an estimated life expectancy of $\geq 6$ months. Patients were considered suitable for the study if they were treated with adjuvant chemotherapy with a curative intent or with first-line chemotherapy for advanced disease. The baseline $\mathrm{Hb}$ levels (prior to chemotherapy initiation) were required to be $>11 \mathrm{mg} / \mathrm{dl}$, to exclude patients with cancer-related anemia at diagnosis. At study entry, patients were also required to have normal folic acid $(>2.5 \mathrm{ng} / \mathrm{ml})$ and vitamin B12 (>200 pg/ml) levels, iron efficiency (transferrin saturation $>15 \%$, ferritin $>50 \mathrm{ng} / \mathrm{ml}$ ) and an Eastern Cooperative Oncology Group performance status of 0-2. Patients with anemia from other causes (e.g., undergoing renal dialysis), as well as patients who had previously received ESAs for any reason or a blood transfusion within 1 month prior to evaluation, were excluded from the study.

The eligible patients were randomized in two groups: Patients in group A (experimental arm) received prophylactic ESA (epoetin $\alpha$, epoetin $\beta$ or darbepoetin $\alpha$, according to the treating physician's choice in standard recommended doses) with iron supplementation (oral or intravenous), with a target $\mathrm{Hb}$ level of $14 \mathrm{mg} / \mathrm{dl}$. If the $\mathrm{Hb}$ levels exceeded this threshold, ESA administration was discontinued and was resumed when $\mathrm{Hb}$ levels were decreased to $<12 \mathrm{mg} / \mathrm{dl}$. In group $\mathrm{B}$, patients received only iron supplementation; ESA administration was initiated only if $\mathrm{Hb}$ levels were decreased to $<11 \mathrm{mg} / \mathrm{dl}$ during chemotherapy and was continued with a target level of $13 \mathrm{mg} / \mathrm{dl}$, at which point ESA support was withheld, only to be resumed when $\mathrm{Hb}$ levels again decreased to $<11 \mathrm{mg} / \mathrm{dl}$. In both groups, $\mathrm{PRBC}$ transfusion was allowed only when $\mathrm{Hb}$ levels were decreased to $<9 \mathrm{mg} / \mathrm{dl}$. ESA administration was to be continued for 4 weeks after cessation of chemotherapy. The study protocol is illustrated in Fig. 1.

The primary endpoint of the study was safety, with emphasis on thrombosis-related adverse events, while the secondary endpoints included progression-free survival and OS. Toxicity was recorded and graded for severity according to the National Cancer Institute Common Toxicity Criteria for Solid Tumors, version 2.0 (http://ctep.cancer.gov/protocolDevelopment/ electronic_applications/docs/ctcmanual_v4_10-4-99.pdf). The clinical protocol was approved by the Hellenic Cooperative Oncology Group (HeCOG) Protocol Review Committee and by the Institutional Review Board of 'Agii Anargiri' Cancer Hospital. All the patients provided written informed consent for the use of their biological material (blood samples). The trial was registered in the Australian New Zealand Clinical Trials Registry and allocated the registration no. ACTRN12614001082695.

Statistical analysis. The HE 30/02 trial was designed to recruit 800 patients in order to assess differences in the primary 


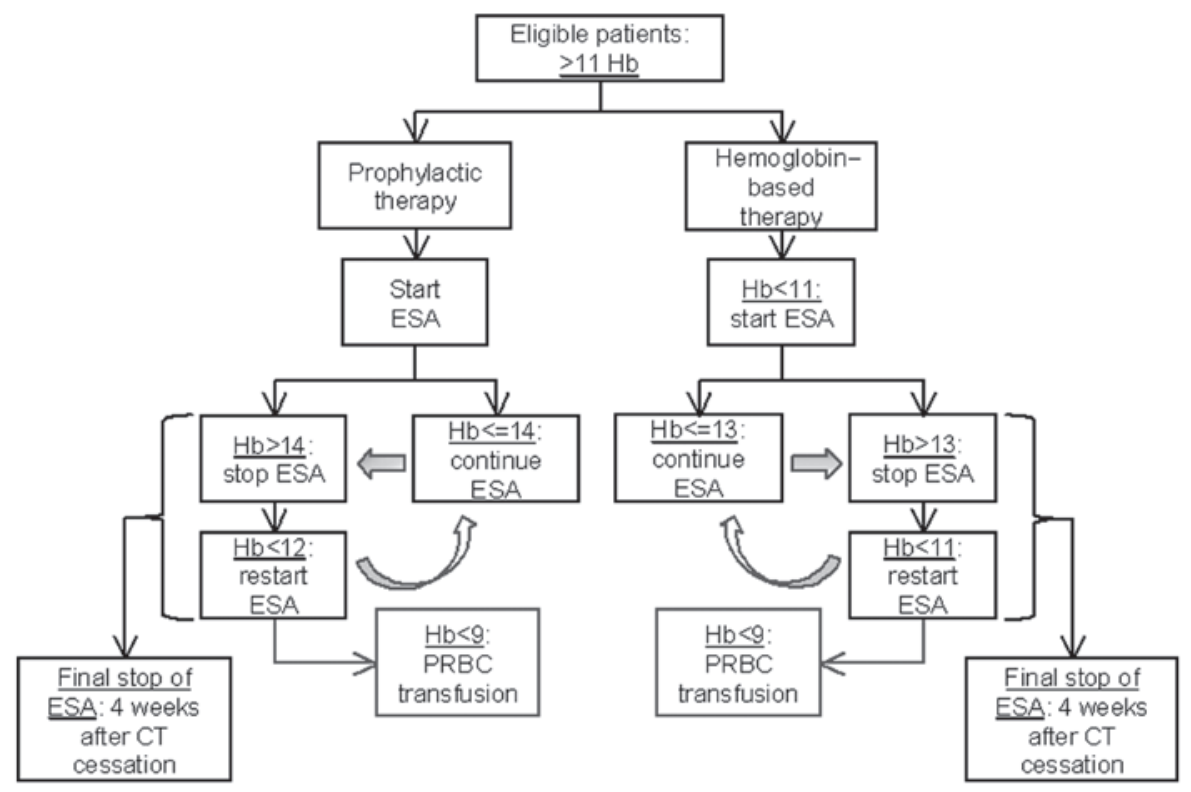

Figure 1. Schematic illustration of the study protocol. Hb, hemoglobin; ESA, erythropoiesis-stimulating agent; PRBC, packed red blood cell; CT, chemotherapy.

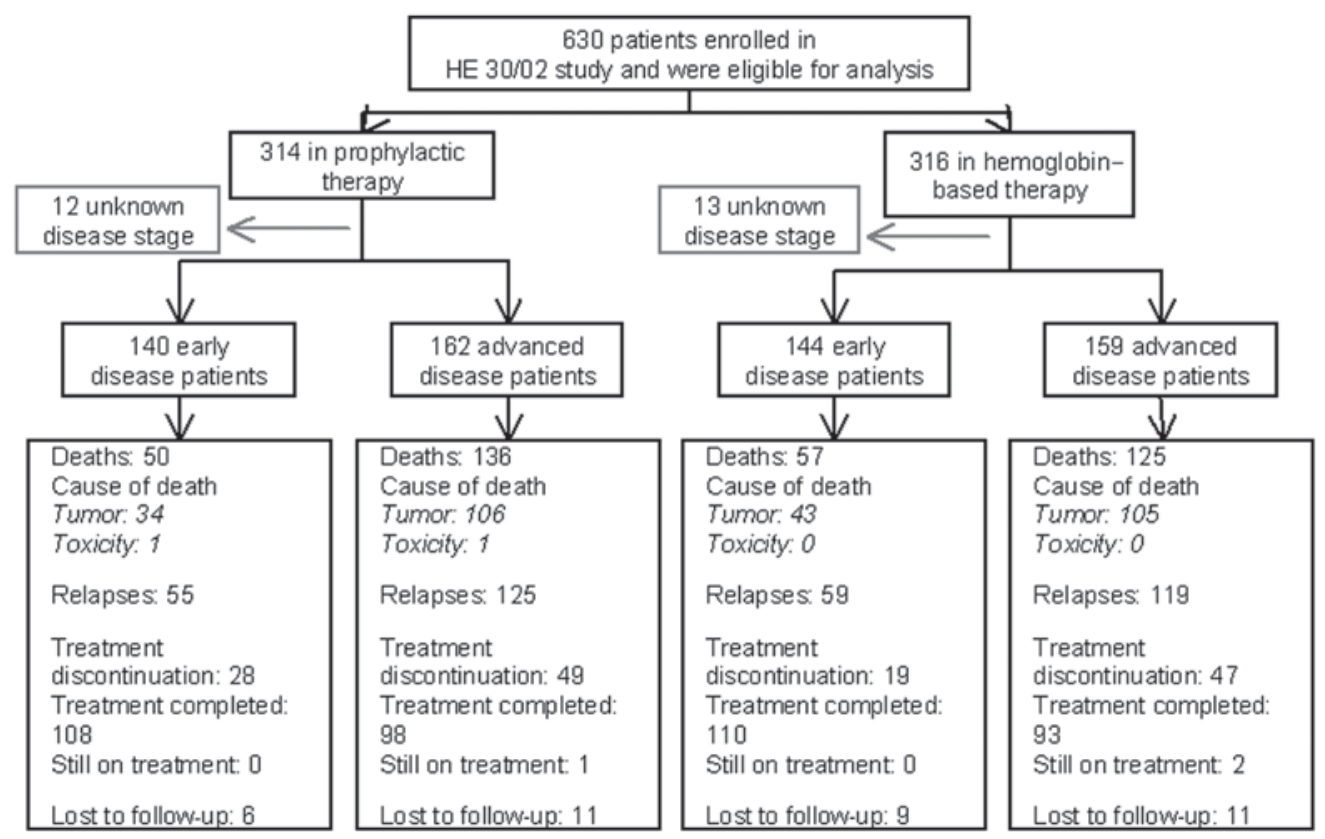

Figure 2. Consort flow diagram of the study.

endpoint of the study (safety) between the treatment arms, but was stopped prematurely due to the FDA recommendation in 2007, with 630 patients already enrolled.

Categorical variables are presented as frequencies and percentages, while various measures (mean, median and range) are used for continuous variables. The associations between categorical variables, such as clinical characteristics and toxicity data among patient cohorts were examined with the Chi-square test and the Fisher's exact test where appropriate.

For patients with advanced disease, OS was measured from the time of initiation of first-line treatment until death from any cause or date of the last contact. For patients with early disease, OS was measured from the date of initiation of adjuvant treatment.

In order to assess the differences in average $\mathrm{Hb}$ levels among the two cohorts, mixed models were used separately for the early- and advanced-disease patients. The models included time in weeks as a polynomial of third degree and an interaction term with the treatment group in order to take into consideration the trend and differences, respectively, in $\mathrm{Hb}$ temporal variation during the treatment period, while the compound symmetry structure was used for covariance matrix.

The survival status was updated in January, 2012. Time-to-event distributions were estimated using Kaplan-Meier 
Table I. Comparison of basic patient characteristics and outcome between prophylactic and Hb-based ESA administration.

A, Patient characteristics

\begin{tabular}{|c|c|c|c|c|c|c|}
\hline \multirow[b]{2}{*}{ Characteristics } & \multicolumn{3}{|c|}{ Early disease } & \multicolumn{3}{|c|}{ Advanced disease } \\
\hline & $\begin{array}{l}\text { Hemoglobin-based } \\
\qquad(\mathrm{n}=144)\end{array}$ & $\begin{array}{l}\text { Prophylactic } \\
\quad(\mathrm{n}=140)\end{array}$ & P-value & $\begin{array}{l}\text { Hemoglobin-based } \\
\qquad(\mathrm{n}=159)\end{array}$ & $\begin{array}{l}\text { Prophylactic } \\
\qquad(\mathrm{n}=140)\end{array}$ & P-value \\
\hline Age, years & & & 0.918 & & & 0.100 \\
\hline Mean (SD) & $59.9(12.1)$ & $60.1(12.0)$ & & $62.3(12.2)$ & $60.1(12.6)$ & \\
\hline Median & 62.20 & 64.30 & & 65.10 & 61.95 & \\
\hline Range & $29-78$ & $29-78$ & & $27-83$ & $20-84$ & \\
\hline BSA & & & 0.274 & & & 0.237 \\
\hline Mean (SD) & $26.8(4.3)$ & $26.3(3.9)$ & & $26.7(5.3)$ & $25.9(4.6)$ & \\
\hline Median & 27.10 & 26.20 & & 25.80 & 25.80 & \\
\hline Range & $16-37$ & $19-37$ & & $15-44$ & $18-46$ & \\
\hline Age (at median), n (\%) & & & 0.402 & & & 0.091 \\
\hline$<63$ & $74(51.4)$ & $65(46.4)$ & & $71(44.6)$ & $87(53.8)$ & \\
\hline$\geq 63$ & $68(47.2)$ & $73(52.2)$ & & $85(53.5)$ & $71(43.8)$ & \\
\hline Not reported & $2(1.4)$ & $2(1.4)$ & & $3(1.9)$ & $4(2.4)$ & \\
\hline Concomitant CRT, n (\%) & & & 0.185 & & & 0.159 \\
\hline No & $97(67.4)$ & $85(60.8)$ & & 125 (78.6) & $111(68.6)$ & \\
\hline Yes & $27(18.8)$ & $35(25.0)$ & & $6(3.8)$ & $11(6.8)$ & \\
\hline Not reported & $20(13.8)$ & $20(14.2)$ & & $28(17.6)$ & $40(24.6)$ & \\
\hline Gender, n (\%) & & & 0.499 & & & 0.183 \\
\hline Male & $53(36.8)$ & $57(40.8)$ & & $60(37.8)$ & $73(45.0)$ & \\
\hline Female & $91(63.2)$ & $83(59.2)$ & & $99(62.2)$ & $89(55.0)$ & \\
\hline Malignacy, n (\%) & & & 0.503 & & & 0.743 \\
\hline Breast & $39(27.1)$ & $43(30.8)$ & & $26(16.4)$ & $22(13.6)$ & \\
\hline Gynaecological & $2(1.4)$ & $0(0.0)$ & & $27(17.0)$ & $31(19.2)$ & \\
\hline Gastric & $8(5.6)$ & $13(9.2)$ & & $9(5.6)$ & $5(3.0)$ & \\
\hline Colorectal & $79(54.8)$ & $74(52.8)$ & & $38(23.8)$ & $36(22.2)$ & \\
\hline Pancreatic/hepatic & $2(1.4)$ & $2(1.4)$ & & $7(4.4)$ & $9(5.6)$ & \\
\hline Prostate & $0(0.0)$ & $0(0.0)$ & & $1(0.6)$ & $0(0.0)$ & \\
\hline Non-prostate GU & $8(5.6)$ & $4(2.8)$ & & $25(15.8)$ & $32(19.8)$ & \\
\hline Lung & $5(3.5)$ & $3(2.2)$ & & $20(12.6)$ & $25(15.4)$ & \\
\hline Not reported & $1(0.6)$ & $1(0.8)$ & & $6(3.8)$ & $2(1.2)$ & \\
\hline PS score (WHO), n (\%) & & & 0.715 & & & 0.464 \\
\hline 0 & $117(81.2)$ & $113(80.8)$ & & $91(57.2)$ & $100(61.8)$ & \\
\hline $1-2-3$ & $19(13.2)$ & $22(15.6)$ & & $57(35.8)$ & $51(31.4)$ & \\
\hline Not reported & $8(5.6)$ & $5(3.6)$ & & $11(7.0)$ & $11(6.8)$ & \\
\hline Transfusion history, n (\%) & & & 0.350 & & & 0.524 \\
\hline No & $111(77.0)$ & $100(71.4)$ & & $121(76.2)$ & $129(79.6)$ & \\
\hline Yes & $14(9.8)$ & $18(12.8)$ & & $8(5.0)$ & $6(3.8)$ & \\
\hline Not reported & $19(13.2)$ & $22(15.8)$ & & $30(18.8)$ & $27(16.6)$ & \\
\hline
\end{tabular}

B, Outcome

Early disease

Characteristics

Hemoglobin-based Prophylactic P-value

Advanced disease

FU, months

Median (range)

$85.0(0.2-111.9)$

$86.6(0.7-107.8)$

$83.8(0.3-94.3)$

$86.3(0.3-102.5)$ 
Table I. Continued.

B, Outcome

\begin{tabular}{|c|c|c|c|c|c|c|}
\hline \multirow[b]{2}{*}{ Characteristics } & \multicolumn{3}{|c|}{ Early disease } & \multicolumn{3}{|c|}{ Advanced disease } \\
\hline & Hemoglobin-based & Prophylactic & P-value & Hemoglobin-based & Prophylactic & P-value \\
\hline OS, months & & & $0.6008^{\mathrm{a}}$ & & & $0.7216^{\mathrm{a}}$ \\
\hline Median $(95 \% \mathrm{CI})$ & NYR (80.8-NYR) & NYR (87.9-NYR) & & $13.4(11.2-16.3)$ & $17.4(13.8-21.8)$ & \\
\hline $\begin{array}{l}\text { Event-free } \\
\text { at } 3 \text { years }(\%)\end{array}$ & 77.3 & 76.3 & & 25.2 & 26.5 & \\
\hline Total events & $57 / 137(41.6 \%)$ & $50 / 131(38.2 \%)$ & & $125 / 148(84.5 \%)$ & $136 / 154(88.3 \%)$ & \\
\hline DFS, months & & & & & & $0.9102^{\mathrm{a}}$ \\
\hline Median (95\% CI) & NYR (71.8-NYR) & NYR (80.8-NYR) & & - & - & \\
\hline $\begin{array}{l}\text { Event-free } \\
\text { at } 3 \text { years }(\%)\end{array}$ & 65.7 & 64.7 & & - & - & \\
\hline Total events & $59 / 141(41.8 \%)$ & $55 / 137(40.1 \%)$ & & - & - & \\
\hline PFS, months & & & & & & $0.4760^{\mathrm{a}}$ \\
\hline Median $(95 \% \mathrm{CI})$ & - & - & & $8.0(7.4-9.7)$ & $10.2(8.9-13.3)$ & \\
\hline $\begin{array}{l}\text { Event-free } \\
\text { at } 3 \text { years }(\%)\end{array}$ & - & - & & 21.2 & 18.3 & \\
\hline Total events & - & - & & $119 / 157(75.8 \%)$ & $125 / 159(78.6 \%)$ & \\
\hline
\end{tabular}

${ }^{a}$ Log-rank test P-value. ESA, erythropoiesis-stimulating agent; NYR, not yet reached; BSA, body surface area; SD, standard deviation; CRT, chemoradiotherapy; GU, genitourinary; PS, performance status; CI, confidence interval; WHO, World Health Organization; FU, follow-up; OS, overall survival; DFS, disease-free survival; PFS, progression-free survival.

curves and the log-rank test was used to examine differences. For all univariate tests, the significance level was set at $\alpha=0.05$. SAS software was used for statistical analysis (SAS for Windows, version 9.3; SAS Institute Inc., Cary, NC, USA).

\section{Results}

Patient and treatment characteristics. Between 2002 and 2007 (year of accrual suspension), 630 patients from 10 comprehensive cancer centers in Greece were randomized in the two study arms and followed up for a median of 85.4 months (range: 0.2-111.9 months). As shown in Fig. 2 (consort diagram), 314 patients had been randomized to receive prophylactic (group A) and 316 patients Hb-based (group B) ESA. In total, 66 patients were either lost to follow-up or had incomplete data; final safety and efficacy analysis was conducted on 605 and 564 patients, respectively. At the time of the data cut-off (January, 2012), 358 patients had relapsed and 380 patients had succumbed to the disease.

The major tumor types included colorectal (36.0\%), breast $(20.6 \%)$, non-prostate genitourinary $(11.0 \%)$ and lung cancer $(8.4 \%)$. Approximately half of the patients (51.0\%) had advanced disease at diagnosis, $40.6 \%$ had $\mathrm{Hb}$ levels $<12 \mathrm{mg} / \mathrm{dl}$ at baseline and $49.8 \%$ received ESAs prophylactically (group A, 1:1 randomization). As shown in Table I, there were no significant differences between groups A and B in basic clinical characteristics for patients who received adjuvant chemotherapy for potentially curable disease, or for those who received first-line chemotherapy for advanced cancer.
Patients received chemotherapy for a median of 18.6 weeks (range: 1-202 weeks). The most commonly used cytotoxic agents were antimetabolites (5-fluorouracil, 44.9\%; gemcitabine, $12.5 \%$; and methotrexate, $8.3 \%$ ), platinum analogs (carboplatin, 21.9\%; cisplatin, 6.8\%; and oxaliplatin, 3.0\%), alkylating agents (cyclophosphamide, $11.3 \%$ ), anthracyclines (epiroubicin, 13.3\%), camptothecins (irinotecan, 21.6\%) and taxanes (paclitaxel, 22.9\%; and docetaxel, 11.4\%).

Response to ESAs. As shown in Fig. 3, prophylactic ESA administration, compared with Hb-based administration, was associated with a significant increase in $\mathrm{Hb}$ levels throughout the first 25 weeks of chemotherapy in the advanced-disease patient cohort and a non-significant (trend) increase in those with early disease $(\mathrm{P}=0.0011$ for patients with advanced disease and $\mathrm{P}=0.0987$ for patients with early disease). Considering the first 12 weeks of treatment, statistical significance was reached in both cohorts $(\mathrm{P}<0.001$ for patients with advanced disease and $\mathrm{P}=0.002$ for patients treated with a curative intent). Of note, the increase in $\mathrm{Hb}$ levels in response to ESA support did not differ significantly between the two treatment groups, although numerically it was more prominent in the group of patients who received chemotherapy with a curative intent (Fig. 3A) compared with those who received chemotherapy for advanced disease (Fig. 3B) $(\mathrm{P}=0.363$ for the first 12 weeks and $\mathrm{P}=0.167$ for the first 25 weeks). During the chemotherapy courses, the Hb levels also started to rise in the Hb-based ESA administration patient group, since longer duration of chemotherapy was associated with an additive myelosuppressive 

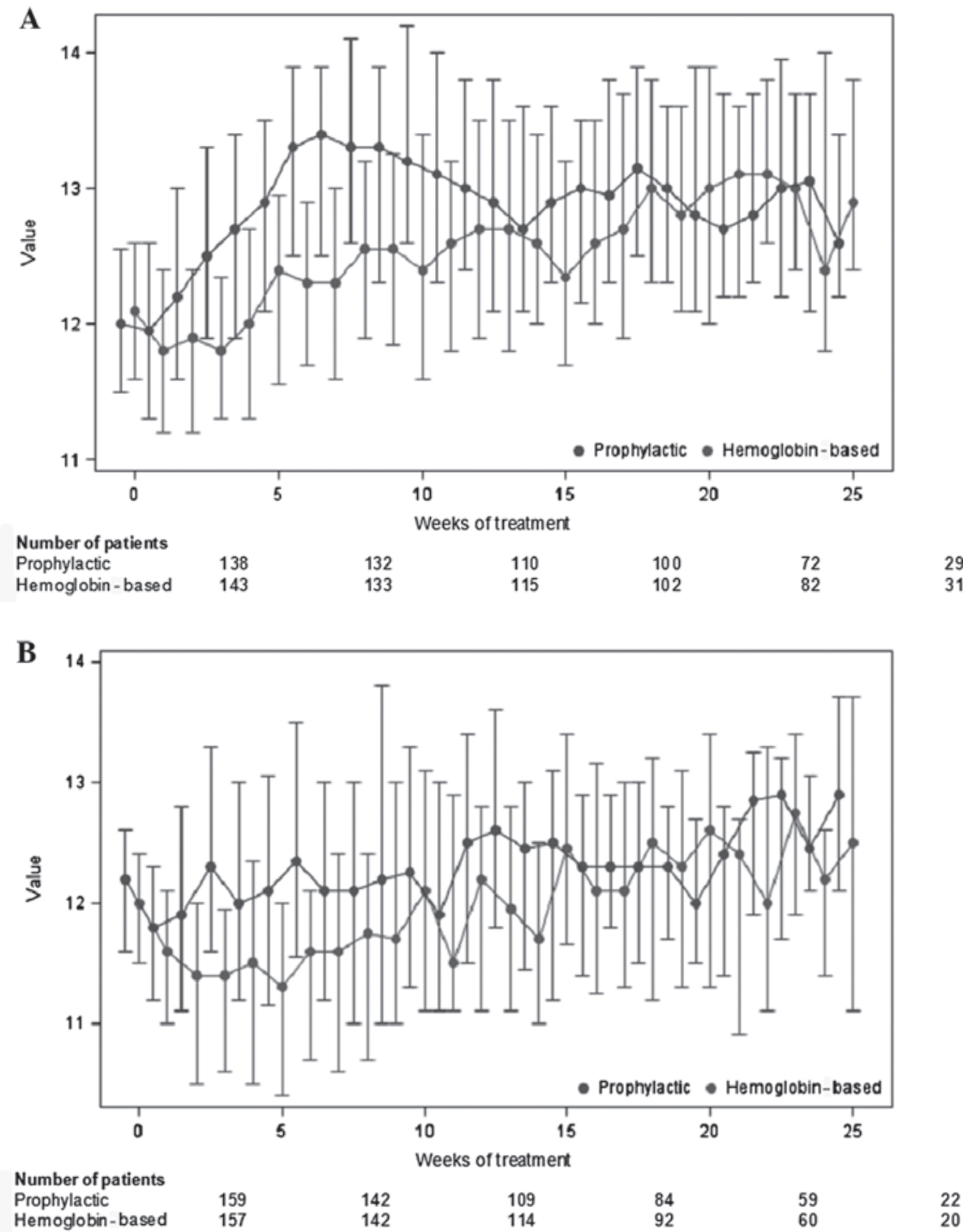

Figure 3. (A) Illustration of the median and quartile values of hemoglobin for patients with early disease. (B) Illustration of the median and quartile values of hemoglobin for patients with advanced disease.

effect and consequent anemia that prompted ESA administration as per protocol, resulting in intercepted curves with time progression (Fig. 3).

Safety and toxicity (primary outcome). Of the 605 patients who were evaluable for safety and toxicity, 284 had received adjuvant treatment with curative intent and 321 had received first-line treatment for advanced disease (Table II). Among patients treated with adjuvant chemotherapy, those who received ESA prophylactically (group A), compared with those who received ESA with an $\mathrm{Hb}$-based algorithm (group B), exhibited a significantly lower incidence of overall (grades 1-4) anemia (2.9 vs. 9.0\%, respectively; $\mathrm{P}=0.0428$ ) and a marginally lower incidence of severe (grades 3-4) anemia ( 0.0 vs. $3.5 \%$, respectively; $\mathrm{P}=0.0604$ ). However, the same patient group exhibited a higher incidence of overall deep venous thrombosis, albeit not at a significant level (all grades: 9.3 vs. $4.2 \%$, respectively; $\mathrm{P}=0.0993$ ); the differences in the incidence of stroke, pulmonary embolism, or myocardial infarction (all grades) between the two groups were not significant $(\mathrm{P}>0.9999, \mathrm{P}=0.3654$ and $\mathrm{P}=0.34$, respectively; data not shown). However, an increased incidence of all grades of a composite outcome encompassing all thrombosis-related events (deep venous thrombosis, pulmonary embolism, myocardial infarction and stroke), was recorded as a non-significant trend (all grades: 12.9 vs. $6.3 \%, \mathrm{P}=0.0692$; grades 3-4: 2.9 vs. $2.1 \%$; $\mathrm{P}=0.7198$ ), (Table IIA).

Among patients treated with first-line chemotherapy for advanced disease (Table IIB), those who received ESA prophylactically, compared with those who received ESA with an $\mathrm{Hb}$-based algorithm, exhibited a significantly lower incidence of overall (grades 1-4) anemia (5.6 vs. $13.8 \%$, respectively; $\mathrm{P}=0.0138$ ) and severe (grades 3-4) anemia (3.1 vs. 8.8\%, respectively; $\mathrm{P}=0.0342$ ), as well as of overall and severe fatigue (all grades: 10.5 vs. $27.7 \%$, respectively, $\mathrm{P}<0.0001$; grades $3-4$ : 3.1 vs. $8.2 \%$, respectively, $\mathrm{P}=0.0545$ ). The same patient group also experienced a non-significant trend for more events of deep venous thrombosis (all grades: $\mathrm{P}=0.2277$ ) and stroke (all grades: $\mathrm{P}=0.1737$ ), but not of pulmonary embolism (all grades: $\mathrm{P}=0.9999$ ) or myocardial infarction (all grades: $\mathrm{P}=0.74$; data 
Table II. Comparison of safety and toxicity between prophylactic and Hb-based erythropoiesis-stimulating agent administration.

A, Patients receiving adjuvant treatment: All-grade and severe (grades 3 and 4) toxicity

\begin{tabular}{|c|c|c|c|c|c|c|c|c|}
\hline \multirow[b]{2}{*}{ Toxicity } & \multicolumn{3}{|c|}{ All grades } & \multirow[b]{2}{*}{ P-value } & \multicolumn{3}{|c|}{ Grades 3-4 } & \multirow[b]{2}{*}{ P-value } \\
\hline & $\begin{array}{c}\text { Overall, } \\
\mathrm{n}=284\end{array}$ & $\begin{array}{c}\text { Prophylactic, } \\
n=140\end{array}$ & $\begin{array}{c}\text { Hb-based } \\
n=144\end{array}$ & & $\begin{array}{c}\text { Overall, } \\
n=284\end{array}$ & $\begin{array}{l}\text { Prophylactic, } \\
n=140\end{array}$ & $\begin{array}{c}\text { Hb-based } \\
n=144\end{array}$ & \\
\hline Anemia & 17 & 4 & 13 & 0.0428 & 5 & - & 5 & 0.0604 \\
\hline Leukopenia & 31 & 15 & 16 & $>0.9999$ & 11 & 7 & 4 & 0.3724 \\
\hline Neutropenia & 26 & 14 & 12 & 0.6838 & 15 & 8 & 7 & 0.7959 \\
\hline Thrombocytopenia & 15 & 9 & 6 & 0.4367 & 5 & 4 & 1 & 0.2090 \\
\hline Stomatitis & 8 & 4 & 4 & $>0.9999$ & 1 & - & 1 & $>0.9999$ \\
\hline Diarrhea & 10 & 4 & 6 & 0.7497 & 2 & 1 & 1 & $>0.9999$ \\
\hline Fatigue & 13 & 4 & 9 & 0.2562 & 3 & - & 3 & 0.2475 \\
\hline Peripheral neuropathy & 11 & 5 & 6 & $>0.9999$ & 1 & 1 & - & 0.4930 \\
\hline Infection & 8 & 5 & 3 & 0.4963 & 1 & - & 1 & $>0.9999$ \\
\hline Deep venous thrombosis & 19 & 13 & 6 & 0.0993 & 2 & 1 & 1 & $>0.9999$ \\
\hline Pulmonary embolism & 4 & 3 & 1 & 0.3654 & 3 & 2 & 1 & 0.6184 \\
\hline Stroke & 4 & 2 & 2 & $>0.9999$ & 2 & 1 & 1 & $>0.9999$ \\
\hline $\begin{array}{l}\text { Thrombosis-related events } \\
\text { (composite outcome) }\end{array}$ & 27 & 18 & 9 & 0.0692 & 7 & 4 & 3 & 0.7198 \\
\hline
\end{tabular}

B, Patients receiving first-line treatment: All-grade and severe (grades 3 and 4) toxicity

\begin{tabular}{|c|c|c|c|c|c|c|c|c|}
\hline \multirow[b]{2}{*}{ Toxicity } & \multicolumn{3}{|c|}{ All grades } & \multirow[b]{2}{*}{ P-value } & \multicolumn{3}{|c|}{ Grades 3-4 } & \multirow[b]{2}{*}{ P-value } \\
\hline & $\begin{array}{l}\text { Overall, } \\
\mathrm{n}=321\end{array}$ & $\begin{array}{l}\text { Prophylactic, } \\
\quad n=162\end{array}$ & $\begin{array}{l}\text { Hb-based } \\
\mathrm{n}=159\end{array}$ & & $\begin{array}{l}\text { Overall, } \\
n=321\end{array}$ & $\begin{array}{l}\text { Prophylactic, } \\
\quad n=162\end{array}$ & $\begin{array}{l}\text { Hb-based, } \\
\mathrm{n}=159\end{array}$ & \\
\hline Anemia & 31 & 9 & 22 & 0.0138 & 19 & 5 & 14 & 0.0342 \\
\hline Leukopenia & 47 & 25 & 22 & 0.7531 & 25 & 14 & 11 & 0.6781 \\
\hline Neutropenia & 40 & 22 & 18 & 0.6133 & 28 & 14 & 14 & $>0.9999$ \\
\hline Thrombocytopenia & 28 & 10 & 18 & 0.1161 & 10 & 3 & 7 & 0.2156 \\
\hline Stomatitis & 13 & 6 & 7 & 0.7845 & 4 & 2 & 2 & $>0.9999$ \\
\hline Diarrhea & 22 & 9 & 13 & 0.3844 & 6 & 2 & 4 & 0.4451 \\
\hline Fatigue & 61 & 17 & 44 & $<0.0001$ & 18 & 5 & 13 & 0.0545 \\
\hline Peripheral neuropathy & 13 & 8 & 5 & 0.5732 & 4 & 2 & 2 & $>0.9999$ \\
\hline Infection & 18 & 8 & 10 & 0.6353 & 5 & 3 & 2 & $>0.9999$ \\
\hline Deep venous thrombosis & 27 & 17 & 10 & 0.2277 & 9 & 6 & 3 & 0.5017 \\
\hline Pulmonary embolism & 9 & 5 & 4 & $>0.9999$ & 7 & 4 & 3 & $>0.9999$ \\
\hline Stroke & 9 & 7 & 2 & 0.1737 & 7 & 6 & 1 & 0.1210 \\
\hline $\begin{array}{l}\text { Thrombosis-related events } \\
\text { (composite outcome) }\end{array}$ & 45 & 29 & 16 & 0.0430 & 23 & 16 & 7 & 0.0990 \\
\hline
\end{tabular}

C, All patients: All-grade and severe (grades 3 and 4) toxicity

\begin{tabular}{|c|c|c|c|c|c|c|c|c|}
\hline \multirow[b]{2}{*}{ Toxicity } & \multicolumn{3}{|c|}{ All grades } & \multirow[b]{2}{*}{ P-value } & \multicolumn{3}{|c|}{ Grades 3-4 } & \multirow[b]{2}{*}{ P-value } \\
\hline & $\begin{array}{c}\text { Overall, } \\
n=605\end{array}$ & $\begin{array}{c}\text { Prophylactic, } \\
n=302\end{array}$ & $\begin{array}{l}\text { Hb-based, } \\
n=303\end{array}$ & & $\begin{array}{c}\text { Overall, } \\
n=605\end{array}$ & $\begin{array}{l}\text { Prophylactic, } \\
\quad n=302\end{array}$ & $\begin{array}{c}\text { Hb-based, } \\
n=303\end{array}$ & \\
\hline Anemia & 48 & 13 & 35 & 0.0014 & 24 & 5 & 19 & 0.0056 \\
\hline Leukopenia & 78 & 40 & 38 & 0.8095 & 36 & 21 & 15 & 0.3087 \\
\hline Neutropenia & 66 & 36 & 30 & 0.4372 & 43 & 22 & 21 & 0.8758 \\
\hline Thrombocytopenia & 43 & 19 & 24 & 0.5273 & 15 & 7 & 8 & $>0.9999$ \\
\hline Stomatitis & 21 & 10 & 11 & $>0.9999$ & 5 & 2 & 3 & $>0.9999$ \\
\hline
\end{tabular}


Table II. Continued.

C, All patients: All-grade and severe (grades 3 and 4) toxicity

\begin{tabular}{|c|c|c|c|c|c|c|c|c|}
\hline \multirow[b]{2}{*}{ Toxicity } & \multicolumn{3}{|c|}{ All grades } & \multirow[b]{2}{*}{ P-value } & \multicolumn{3}{|c|}{ Grades 3-4 } & \multirow[b]{2}{*}{ P-value } \\
\hline & $\begin{array}{c}\text { Overall, } \\
\mathrm{n}=605\end{array}$ & $\begin{array}{l}\text { Prophylactic, } \\
\quad \mathrm{n}=302\end{array}$ & $\begin{array}{l}\text { Hb-based } \\
\text { n=303 }\end{array}$ & & $\begin{array}{l}\text { Overall, } \\
\mathrm{n}=605\end{array}$ & $\begin{array}{l}\text { Prophylactic, } \\
n=302\end{array}$ & $\begin{array}{l}\text { Hb-based } \\
\mathrm{n}=303\end{array}$ & \\
\hline Diarrhea & 32 & 13 & 19 & 0.3640 & 8 & 3 & 5 & 0.7247 \\
\hline Fatigue & 74 & 21 & 53 & $<0.001$ & 21 & 5 & 16 & 0.0241 \\
\hline Peripheral neuropathy & 24 & 13 & 11 & 0.6837 & 5 & 3 & 2 & 0.6859 \\
\hline Infection & 26 & 13 & 13 & $>0.9999$ & 6 & 3 & 3 & $>0.9999$ \\
\hline Deep venous thrombosis & 46 & 30 & 16 & 0.0325 & 11 & 7 & 4 & 0.3829 \\
\hline Pulmonary embolism & 13 & 8 & 5 & 0.4184 & 10 & 6 & 4 & 0.5451 \\
\hline Stroke & 13 & 9 & 4 & 0.1745 & 9 & 7 & 2 & 0.1065 \\
\hline $\begin{array}{l}\text { Thrombosis-related events } \\
\text { (composite outcome) }\end{array}$ & 72 & 47 & 25 & 0.0057 & 30 & 20 & 10 & 0.0636 \\
\hline
\end{tabular}

not shown) resulting in a marginally significant increase in the incidence of the composite outcome of all thrombosis-related events (all grades: 17.9 vs. $10.1 \%, \mathrm{P}=0.043$, grades $3-4$ : 9.9 vs. $4.4 \%, \mathrm{P}=0.099)$.

Notably, the aforementioned differences were more prominent in the group of patients who were treated with first-line chemotherapy for advanced disease, compared with those who were treated with adjuvant chemotherapy with a curative intent; this observation was consistent throughout the main adverse events associated with thrombosis (deep venous thrombosis, stroke, myocardial infarction and pulmonary embolism) (Table II).

Efficacy. At the time of data cut-off (January, 2012), 358 patients had relapsed and 380 patients had succumbed to the disease. In the whole study cohort and in the per-protocol treated patients, there were no significant differences in clinical outcomes (disease or progression-free and overall survival) with respect to the presence or absence of anemia at study entry and the administration of ESA in a prophylactic or Hb-based manner. The percentages of patients that were event-free at 3 years did not differ (65.7 vs. $64.7 \%)$. The median OS in the group of patients who received adjuvant treatment with a curative intent has not yet been reached [95\% confidence interval (CI): 87.9 months-not estimated] for those who received ESA prophylactically (95\% CI: 80.8-not estimated) or those who received ESA in a Hb-based manner (log-rank test $\mathrm{P}=0.6008$, Fig. 4A). The median OS in the group of patients who received chemotherapy for advanced disease was 17.4 months (95\% CI: 13.8-21.8) for those who received ESA prophylactically and 13.4 months (95\% CI: 11.2-16.3) for those who received ESA in a Hb-based manner (log-rank test $\mathrm{P}=0.7216$, Fig. 4B).

\section{Discussion}

Following an initial peak, prophylactic ESA administration was subsequently halted by the medical community on the grounds
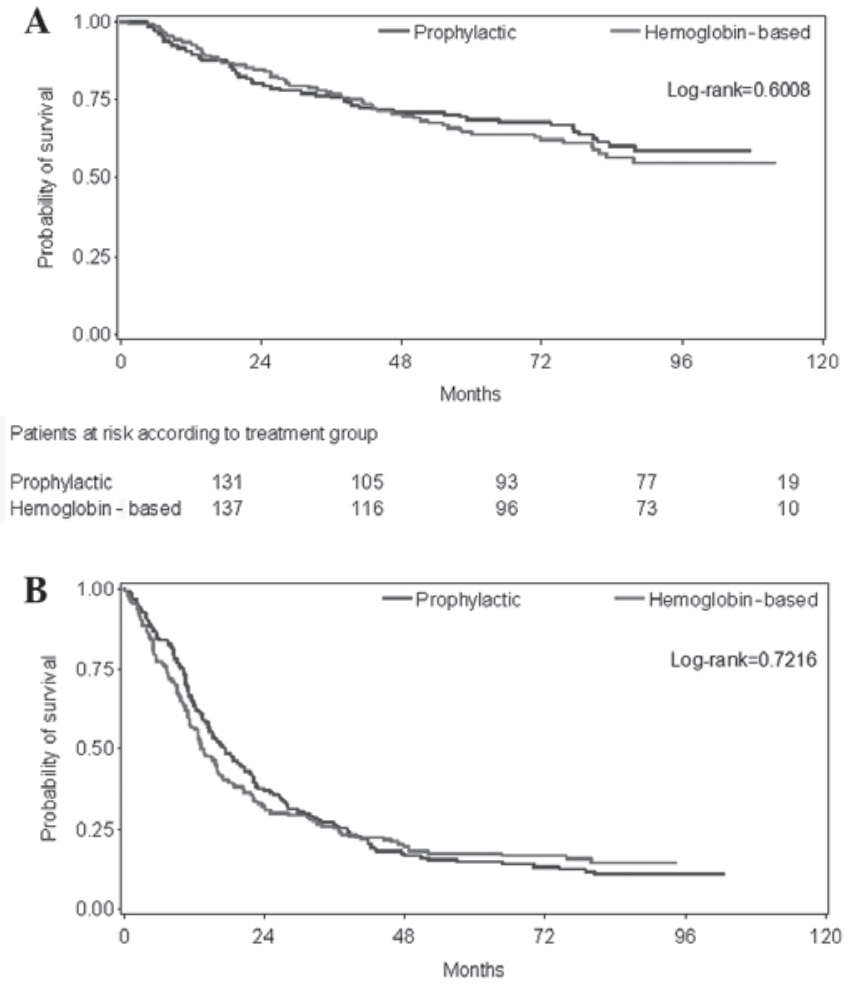

Patients at risk according to treatment group

$\begin{array}{llllcc}\text { Prophylactic } & 151 & 56 & 26 & 18 & 1 \\ \text { Hemoglobin-based } & 146 & 46 & 26 & 21 & 10\end{array}$

Figure 4. (A) Kaplan-Meier curves of overall survival for patients with early disease by study group. (B) Kaplan-Meier curves of overall survival for patients with advanced disease by study group.

of the results reported by various randomized controlled trials in a number of solid and lymphoid malignancies (2-8), which raised concerns regarding safety, including an increase in the incidence of thromboembolic events, disease progression and death. These concerns resulted in the premature termination of ongoing clinical trials at that time, such as the present study. 
However, the long-term follow-up of patients who had been enrolled in these studies provides a unique opportunity to evaluate the safety and toxicity parameters of prophylactic ESA administration and to confirm or to challenge the conclusions of previous studies with a shorter follow-up that suggested a detrimental effect of prophylactic ESA administration on clinical outcome. With a median follow-up of $>7$ years and with $>600$ randomized patients, the present study provides an appropriate framework for the evaluation of long-term toxicity and efficacy outcomes in these patients.

We confirmed that prophylactic ESA administration, as compared to $\mathrm{Hb}$-based, was associated with a significant increase (mean change) in the median $\mathrm{Hb}$ values, as well as a significant reduction in the incidence of CIA, both in patients with early disease receiving adjuvant chemotherapy with a curative intent and in patients with advanced solid tumors receiving first-line chemotherapy. In the group of patients with advanced disease, in particular, prophylactic ESA administration was associated with a significant decrease in the incidence of overall and severe treatment-related fatigue, which is a frequent and disabling symptom in cancer patients. However, prophylactic ESA administration was associated with a statistically non-significant increase in the composite outcome of thrombosis-related adverse events, comprising deep venous thromboembolism, pulmonary embolism, myocardial infarction and stroke. In the group of patients with advanced cancer, in particular, this increase in thrombosis-related events was marginally non-significant (all grades: $\mathrm{P}=0.0533$ ) but may be considered clinically relevant, given that thrombosis-related adverse events represent a major cause of morbidity in cancer patients (1). These results are consistent with previous reports from randomized trials (5-7), which suggested a significant increase in thrombosis-related adverse events in patients who received prophylactic ESAs and contribute to the mount of evidence that raise a safety signal for a hazardous effect of prophylactic ESA administration in terms of CIA. It may be hypothesized that, had the trial not been suspended in 2007 and a larger number of patients had been randomized, a higher number of thrombosis-related adverse events would have occurred and the aforementioned differences would have most likely become statistically significant in our study as well. The higher incidence of thrombosis-related adverse events in these patients was anticipated, since raising $\mathrm{Hb}$ at a target of $14 \mathrm{mg} / \mathrm{dl}$ may lead to increased blood viscosity and erythrocyte aggregation, resulting in a hypercoagulant state. Moreover, the increased incidence of thrombosis-related adverse events in patients receiving first-line chemotherapy for advanced disease, compared with those receiving adjuvant treatment, was also anticipated, since advanced cancer has been consistently reported to predispose to thrombosis $(1,2)$. However, no statistically significant increase in the incidence of deep venous thrombosis, myocardial infarction, stroke or pulmonary embolism was observed in the present study, each one considered as a separate variable.

We were unable to detect any detrimental effect of prophylactic ESA administration in patients with early or in those with advanced disease, as suggested by previous reports (5-7). In the present study, there were no significant differences in terms of OS, disease recurrence or progression rates between the two patient groups. The possible reasons for this discordance include the relatively small size of our cohort, due to the fact that the enrolment of patients was prematurely terminated and the heterogeneity of the study population, which comprised patients with both early and advanced cancer, as well as a variety of tumor types with diverse prognosis, which may have biased clinical outcome comparison. However, our study is strengthened by the long patient follow-up, which allowed the evaluation of late toxicities, such as cardiovascular and other thrombosis-related adverse events. This long-term follow-up enabled a more accurate assessment of the high incidence of thrombosis-related adverse events that were suggested by earlier studies including a larger number of patients but with shorter follow-up $(9,10)$.

The current guidelines do not recommend prophylactic ESA administration for CIA in patients treated with adjuvant chemotherapy with a curative intent, or in patients with normal $\mathrm{Hb}$ levels treated with first-line chemotherapy for advanced cancer. Our results are in line with these recommendations, confirming the potential hazardous role of prophylactic ESA administration on a number of thrombosis-related adverse outcomes. Hb-based ESA administration is currently recommended by most guidelines, although the target $\mathrm{Hb}$ levels may differ among various recommendations. Despite these differences, Hb-based ESA administration is associated with a significant improvement in median $\mathrm{Hb}$ levels and fatigue, and a significant reduction in the incidence of CIA and blood transfusion requirements compared with placebo, without an associated increase in the incidence of thrombosis-related events, and should be considered as the treatment of choice according to the ESMO guidelines.

In conclusion, the results from the long-term follow-up of patients with early or advanced cancer who had received prophylactic ESAs for CIA, suggest a beneficial effect in reducing the severity of anemia and treatment-related fatigue, but also confirm their potential hazardous effect on the incidence of thrombosis-related adverse events. This effect was more prominent in patients who received first-line chemotherapy for advanced cancer, compared with those who received adjuvant chemotherapy for potentially curable disease.

\section{Acknowledgements}

This study was supported by an internal Hellenic Cooperative Oncology Group research grant (HE R_EPO_02).

\section{References}

1. Knight K, Wade S and Balducci L: Prevalence and outcomes of anemia in cancer: A systematic review of the literature. Am J Med 116 (Suppl 7A): 11S-26S, 2004.

2. Smith RE Jr, Aapro MS, Ludwig H, Pintér T, Smakal M, Ciuleanu TE, Chen L, Lillie T and Glaspy JA: Darbepoetin alpha for the treatment of anemia in patients with active cancer not receiving chemotherapy or radiotherapy: Results of a phase III, multicenter, randomized, double-blind, placebo-controlled study. J Clin Oncol 26: 1040-1050, 2008.

3. Clark O, Adams JR, Bennett CL and Djulbegovic B: Erythropoietin, uncertainty principle and cancer related anaemia. BMC Cancer 2: 23, 2002.

4. Bohlius J, Langensiepen S, Schwarzer G, Seidenfeld J, Piper M, Bennett $\mathrm{C}$ and Engert $\mathrm{A}$ : Recombinant human erythropoietin and overall survival in cancer patients: Results of a comprehensive meta-analysis. J Natl Cancer Inst 97: 489-498, 2005. 
5. Littlewood TJ, Bajetta E, Nortier JW, Vercammen E and Rapoport B; Epoetin Alfa Study Group: Effects of epoetin alfa on hematologic parameters and quality of life in cancer patients receiving nonplatinum chemotherapy: Results of a randomized, double-blind, placebo-controlled trial. J Clin Oncol 19: 2865-2874, 2001.

6. Hedenus M, Adriansson M, San Miguel J, Kramer MH, Schipperus MR, Juvonen E, Taylor K, Belch A, Altés A, Martinelli G, et al; Darbepoetin Alfa 20000161 Study Group: Efficacy and safety of darbepoetin alfa in anaemic patients with lymphoproliferative malignancies: A randomized, double-blind, placebo-controlled study. Br J Haematol 122: 394-403, 2003.

7. Henke M, Laszig R, Rübe C, Schäfer U, Haase KD, Schilcher B, Mose S, Beer KT, Burger U, Dougherty C, et al: Erythropoietin to treat head and neck cancer patients with anaemia undergoing radiotherapy: Randomised, double-blind, placebo-controlled trial. Lancet 362: 1255-1260, 2003.
8. Leyland-Jones B, Semiglazov V, Pawlicki M, Pienkowski T, Tjulandin S, Manikhas G, Makhson A, Roth A, Dodwell D, Baselga J, et al: Maintaining normal hemoglobin levels with epoetin alfa in mainly nonanemic patients with metastatic breast cancer receiving first-line chemotherapy: A survival study. J Clin Oncol 23: 5960-5972, 2005.

9. Thomas G, Ali S, Hoebers FJ, Darcy KM, Rodgers WH, Patel M, Abulafia O, Lucci JA III and Begg AC: Phase III trial to evaluate the efficacy of maintaining hemoglobin levels above $12.0 \mathrm{~g} / \mathrm{dl}$ with erythropoietin vs above $10.0 \mathrm{~g} / \mathrm{dl}$ without erythropoietin in anemic patients receiving concurrent radiation and cisplatin for cervical cancer. Gynecol Oncol 108: 317-325, 2008.

10. Wright JR, Ung YC, Julian JA, Pritchard KI, Whelan TJ, Smith C, Szechtman B, Roa W, Mulroy L, Rudinskas L, et al: Randomized, double-blind, placebo-controlled trial of erythropoietin in non-small-cell lung cancer with disease-related anemia. J Clin Oncol 25: 1027-1032, 2007. 